\title{
Modélisation numérique d'écoulements partiellement cavitants dans les turbomachines
}

\author{
par F.X. Catelan, I: Maître \\ Iaboratoire des Ecoulements Géophysiques et Industricls, Grenoble \\ P. Morel \\ Société Europécnne de Propulsion
}

\section{E INTRODUC'IION}

La nécessité de construire des turbomachines de plus en plus perfonnantes el plus compactes done moins onéreuses motive depuis plusieurs decennies les recherches dards ce domaine. Une des principales contraintes limitant l'augmentation des vitesses de rotation est le phénomène de cavitation qui se développe quand la pression est abaissée au niveau de la pression de vapeur saturante. La vaporisation du fluide apparaît principalement dans les zones où la géométría présente de fortes courbures, conme an bord dattaque des aubages où se développent parfois des poches de vapeur ainsi que dans les régions où le fluide est soumis à une vorticitê importante.

La présence de la cavitation au scin d'un système génère dilférents problèmes dont le plus connu et certainement le plus gênant est. te phénomène d'érosion des matériaux pour un fonctionnement cavitant prolongé. Mais clic provoque égalcment des chutes de performances des machines toumantes lorsque le volume de vapeur devient important.

Si l'apparition de la cavitation dans une uurbomachine n'est pas directement signe de chute de performance, le volume de vapeur doil toutefois rester limité. Cela demande done une mâ. trise st surtout une connaissance des difTérents régimes de fone. tionnement de la machine. La simulation numérique des régimes cavilants et la prévision des conditions de perte de performances représentent une approche intértssarte qui permet d'agir en amont au niveau de la conception. Le code cavitant tridimensionnel, CALECHE-MOCA3D, a été développé pour atteindre ces objectifs.

\section{LE CODE CALECHE-MOCA3D}

L'utilisation des modèles tridimensionncls visqueux est limitée dans le milieu industricl où ils sont, le plus souven, employés uniquement dans la dernière phase de validation d'un prototype. La délinition précjse de la géométrie, la construction des maillages avec les raffinements adécuats, les temps de calcul et les outils complexes nécessaires au post-traitement sont trop contraignants pour être insérés dans une chaîne de dimen1. sionnement des machincs hydrauliques.

Si on considère une machine travaillant près de son point de fonctionnement nominal, les effets visqueux, confinés à la proximité immédiate des parois, tr'entraînent pas de modifičà-

\begin{tabular}{|c|c|}
\hline \multicolumn{2}{|c|}{ NOTATIONS } \\
\hline Q: & débit $\left(\mathrm{m}^{3} / \mathrm{s}\right)$ \\
\hline Qn : & débil nominal $\left(\mathrm{m}^{3} / \mathrm{s}\right)$ \\
\hline$\rho:$ & masse volumique $\left(\mathrm{kg} / \mathrm{m}^{3}\right)$ \\
\hline$V_{7}:$ & vitessc débitante $(\mathrm{m} / \mathrm{s})$ \\
\hline W: & vitesse relative $(\mathrm{m} / \mathrm{s})$ \\
\hline$W^{312}:$ & $\begin{array}{l}\text { vilesse relative calculée par } \\
\text { le module CALECHE (n/s) }\end{array}$ \\
\hline$W^{\prime}=W \cdot W^{30}:$ & $\begin{array}{l}\text { vitesse relative de perturba- } \\
\text { tion }(\mathrm{m} / \mathrm{s})\end{array}$ \\
\hline$\Psi^{\prime}:$ & $\begin{array}{l}\text { fonction de courant de } \\
\text { perturbation }\end{array}$ \\
\hline \multicolumn{2}{|c|}{ 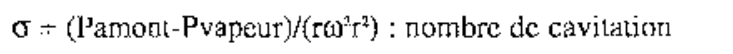 } \\
\hline$\sigma^{*}:$ & $\begin{array}{l}\text { sigma correspondant à un } \\
\text { abaissement de } 3 \% \text { de lá } \\
\text { hautcur manométrique }\end{array}$ \\
\hline R : & rayon $(m)$ \\
\hline $\mathrm{m}$ : & abscisse curviligne \\
\hline 0: & dircction azimutale \\
\hline I : & direction radiale \\
\hline$z:$ & direction axiale \\
\hline
\end{tabular}

Lion majeure de l'écoulement. La modélisation peut alors se faire sur la base d'une résolution tridimensionnelle des équations d'EULER.

Le code développé est donc basé sur unc formulation EULLR-3D (CALECHE, [1]), à laquelle est couplée unc modélisation tridimensionnclle (MOCA3D [2] [3]) des poches de cavitation partielle issue des modèles bidimensionnels [A]. I.a poche de cavitation étant traitée comme une perturbation de l'écoulement non cavitant, un couplage entre le module $3 \mathrm{D}$ el le module cavitant est indispersable. A chaque itération, la poche de cavitation nouvellement calculée par MOCA3D agit comme un corps solide qui forme une sur ćpaisseur à la pale et qui réduit l'espace destiné au fluide dans le canal inter-aubes. I.e calcul suivant, effectué par CALECHE, nernel de réactualiser le champ de vitesses dans le nouveau domaine de calcul qui cortespond à l'espace inter-aubes récl diminué du volume de la poche. Cette 


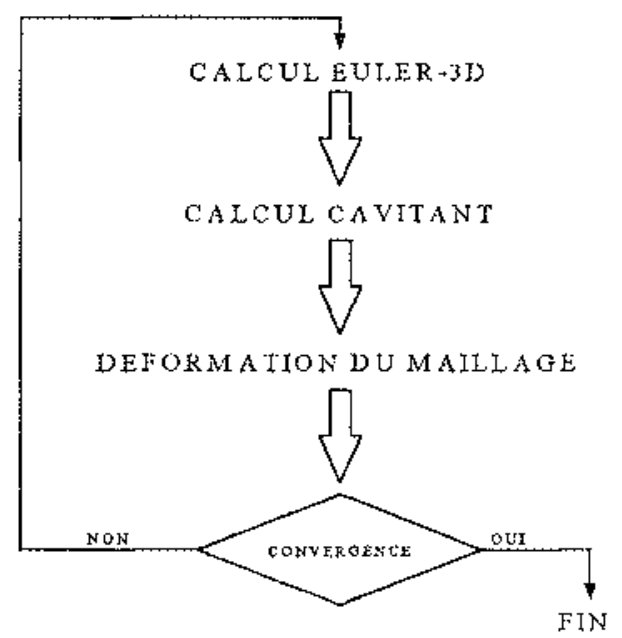

\section{Organigramme général du code CALECHE-MOCA3D.}

interaction qui est à l'origine du processus itératif (Jigure 1) donne à convergence une modélisation de l'écoulement correspondant aux conditions de fonctionncment imposées en début de calcul par l'utilisateur.

\subsection{Modélisation de l'écoulement subcavitant (le code CAI.ECHE-MOCA3D)}

Ce code basé sur une formulation EULFR-3D, a ćté développé par le laboratoire MFTRAFLU sous l'impulsion de l'Electricité de France [1]. Son originalitić provient de l'ulilisation d'une formulation de Clebsch des ćquations d'ELLLER, qui décompose le vec. teur vitesse absolue en une partic potentielle et une partie rotationnelle. L'utilisation de certaines hypothèses simplificatrices permet notamment de se restreindre à la formulation de ClebschHawthorme qui diminue le nombre de variables du problème.

\section{Tं 2.2 Le modèle numérique de cavitation}

[ $\mathcal{c}$ modèle utilisé, adapté à la représentation de la cavitation partielle, est issu du développement de codes bidimensionnels et quasi-tridimensionne]s [4]. Il repose sur une formulation simplifiese (modèle à interface) dont les idées principales sont :

- I a vapcur cst séparéc da liquide par une surface imperméable stationnaire.

- Sur tes trois-quarts avant de la poche de cavitation, la pression est ćgale à la pression de vapeur saturanté.

- Sur le quart arrière, le collapse des structures de vapeur est simulé par urı modèle de sillage.

\subsection{Formulation du problème}

Dans notre modélisation, la construction de la surface dćlimitant la poche de cavitation est le résultat d'une succession de calculs bidimensionnels sur chacune des surfaces axisymétriques constituant le maillage complet de la machine. Cela né constitue qu'une procédure de calcul étanı donné que la modélisation prend en comple, de façon rigoureuse, les effets tridimensionnels par l'intermédiaire des conditions aux linites.
Au couts de l'étape cavilante, la divergence et le rotationnel du champ en projection sur la surface de calcul seront figés. Cette hypolhèse se justinie par un rapport faible cnttc le volume de vapeur et le volume global du canal inter-aubes, exprimant le fait que la cavité ne perturbe que très localement l'écoulement de la machinc. Ces hypothèses de conservation, qui ne sont pass restrictives du fait de lá procédure itérative de la rćsolution, se traduisent par les équations suivantes :

$$
\begin{aligned}
& \operatorname{rot}(\bar{W})=\operatorname{rot}\left(\bar{w}^{3 D}\right) \\
& \operatorname{div}(\bar{W})=\operatorname{div}\left(\bar{w}^{3 D}\right)
\end{aligned}
$$

Dans le but d'alléger la formulation el d'accélérer la convergence des calculs, nous avons raisonné en tenme de perturbation. Catte demière correspond aux modifications causces par la présence de la cavité ; d'où l'introduction d'une vitesse relative $W^{\prime}$ dite de perturbalion définie comme la différence entre les vilesses relatives cavitantes et subcavilantes à une itération donncé. Par conséquent, on peut introduire une fonction de courant. de perturbation $\psi$ ' qui doit être solution de l'équation de Laplace :

$$
\Delta y^{\prime \prime}=0
$$

La fonction de courant de perturbation solution de cette équation est obtenue par une méthođe des éléments finis afin de res. ter cohérent avec le module CALECHE.

\subsubsection{Les conditions aux limites du calcul cavitant}

L'utilisation d'une formulation en perturbation simplific considérablement les conditions aux limites du calcul cavitant. la frontière du domaine de cálcul est découpée en trojs zones : 1. La condition de cavité qui est une condition de Neumann s'exprime sous la forme suivante:

$$
\frac{\partial \Psi^{\prime \prime}}{\partial \mathrm{n}}=\bar{W}^{\prime}(\mathrm{M}) \cdot \overrightarrow{\mathrm{t}}=\vec{W}(\mathrm{M}) \cdot \overrightarrow{\mathrm{t}}-\overline{\mathrm{W}}^{3 \mathrm{D}}(\mathrm{M}) \cdot \overrightarrow{\mathrm{t}}
$$

Cela permet d'exprimer le fait que la vitesse de perturbation est purement tangentielle à l'interface liquide-vapeur. En utilisant les résultats du calcul Euler tridimensionnel, notamment la vilesse relative et la rothalpie, on peut exprimer celle condition en fonction de la vitesse tangenticlle au point de détachement W(D) :

$$
\frac{\partial \Psi^{\prime}}{\partial \mathrm{n}}=\mathrm{C} \overline{\mathrm{W}}(\mathrm{D}) \cdot \overline{\mathrm{t}}-\overline{\mathrm{W}}^{3 \mathrm{D}}(\mathrm{M}) \cdot \overline{\mathrm{t}}
$$

2. La condition de sillage : il est imposé un raccord quadratique à la fonetion de courant de perturbation sur une zone de longueur arbitraire. Nous utiliserons une longueur de sillage qui représente le tiers de la zone à pression constantc ; cette proportion est en bon accord avec les résultats expérimentaux.

3. La zone extéricurc à la poche de cavitation. Dans cettc région il trexiste pas de phénomène perturbant l'écoulement non cavitant, ce qui se traduit par l'annulation de la fonction de courant de perturbation dans cette zone.

\subsubsection{Recherche de la longueur de cavité}

La difficulté de ce type de modélisation est qu'a priori les points d'application des conditions aux limites ne sont pas 
connus. Pour débuter le calcul il faul sc donner une "géométrie de déparr", sachant que le point de détachement " $D$ " est fortement lić à l'ćcoulement au niveru du bord d'attaque de l'aubage cl à l'étz: de la couche limite dans cette région, et que la longueur de la cavité est fonction des répartitions de pression sur l'aube. Pour développer un code prédiclif, nous avons mis en place une méthode d'cstimation de la longueur de la cavité correspondani au niveau de pression en amont de la machine, qui repose sur un balayage de la lo: "L $(\sigma)$ " par dichotomie.

\subsubsection{Déformation du maillage}

Ea recherche de la position de l'interface séparant l'écoulement extérieur de l'intérieur de la cavité est assurée par la conservation du débit initial sur chaque ligne iso-M du maillage.

\subsection{Modélisation du régime critique d'une turbomachine}

La chute de performance d'une machine hydraulique est caractérisćc par son sigma critique (noté $\sigma^{*}$ ). Cet état correspond à une cavitation très développéc quti s'étend at-delà du col ct qui modific le triangle des vitesses en sortie de roue provoquant une chute des performances de la machine. Pour les constructeurs, le tégime critioue correspond, le plus souvent, à un abaissement de $3 \%$ de la hauteur manométrique. Du point de vue numérique, ie plus simple consiste à considérer que le sigma critique comespond à une poche de cavitation qui atteint le recouvrement. Cela représentera donc notre critère numérique pour déceler un régime critique.

Lorsque l'on approche du blocage, la cavitation modifie sensiblement l'écoulement général, ce qui perturbe fortement le couplage "3D-cavilan!". Par conséquenı, pour modéliser ce fonctionncment cxtrême, nous réalisons une "descente en sigma" qui s'apparente à unc procédure expérimentale. Łe calcul est alors réalisé cn plusieurs élapes avec un sigma évoluant du fonctionnement non cavitant au régime critique.

\section{III 늘 PROBLÈME SPÉCIFIQUE AL DÉTACHEMENT}

Les simulations numériques d'écoulements cavitants, notamment avec un modèle è interface, nécessitent le positionnement du point de détachement de la cavité dont dépendent fortement les résultats des modélisations. Lorsque la géométrie considérée présente une arête vive au niveau du bord daltaque, nous savons que la cavitation prendra naissance sur cetle discontinuité ; mais pour les aubages dont l'extrémité est artondie, la position exacte du détachement dies poches de vapeur est numériquement plus délicate à obtenir. Le nombrc de publications liées à ce sujet cst probablement unc preuve de cette difficulté $([5],[6])$.

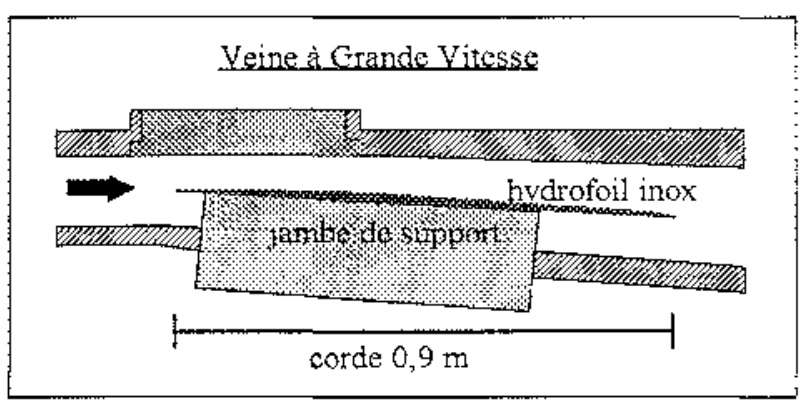

2. Schéma du dispositif expérimental.
De plus, ce positionnement est fortement lić à la discrétisation utilisée pour le bord d'attaque de l'aubage ; pour les géométries tridimensionnelles, te raffinement dis maillage coûte cher en temps de calcul et en place mémoire. Par conséquent, notre objectif est d'étudier la sensibilité des calculs cavitants à la définition géométrique du bord d'attaque des profils, dans le but de supprimer le problème du positionnement du point de détachement (lout en réalisant une discrétisation économique). Pour cela nous utiliscrons la démarche suivante :

1. Adaptation du code CALECHE-MOCA3D pour le calcul de configurations bicimensionnelles (hydrofoils).

2. Utilisation de résultats expérimentaux obtenas sur deux hydrofoils differant par leur bord d'altaque.

3. Comparaisons avec les résuleats d'un code bidimensionnel đéjà validé auparavant.

\section{䋊 3.1 Les essais dans la veine à grande vitesse}

Le projet expérimental [7] est réalisé sur deux géométries de type hydrofoil placées dans une veine disponible au laboratoire (La Veine à Grande Vitesse : V.G.V.). Pour valider le code sur unc géométrie bidimensionnelle, il est nécessaire d'uliliser une géométrie qui ne présente aucune ambiguilté sur la position du détachement des cavités. La seconde géométric constitue un support expérimental nécessaire pour progresser sur le problène de la discrétisation des profils à bord d'attaque pointu : cet hydroloil est assez cffilé et son extrémícé amont est décrite par un arrondi "d'usinage" de $4 / 10^{\text {ime }}$ de millimètre.

Les formes des poches de cavitation sont obtenues par traitement informatique des images vidéo enregistrées lors des essais avec une caméra CCD. Les niveasx de pressions sont estimés soil par les prises pariétales soit par des sondes à quatre trous. Enfin, Ies yitesses près du bori d'allaque sont mesurées par vélociméric laser.

\subsection{Comparaisons calculs - expériences}

Le calcul de l'écoulement cavitant autorr des profils, par le code CALECHE-MOCA3D, est réahisć à l'aide dun maillage construit avec une seule maille en largueur et 3036 éléments irilinéaires soit 31275 nouds. Le raffinement est important dans les zones sensibles comme lc bord d'altaque du profil, le bord de fuite, mais ćgatement près çe l'exurados et de l'intrados du profil

Les comparaisons sont donc téalisées avec les résultats expérimentaux mais également avec un code bidimensionnel qui peut prendre en compte les effets visqueux par une méthode dobstruction, ce qui permettra d'expliquer plus précisément les éventuels décalages.

\subsubsection{Modélisation de l'écoulement cavitant autour du profil biseauté (validation de la version veine)}

Les tendances observées expérimentalement (décroissance de l'épaisseur relative des poches avec leur allongement par exemple) sont reproduites par les calculs. Les formes de poches prédites par les deux codes sont similaires (figure 3) mais souvent plus volumineuses que celles obtenues expérimentajement. La différence provient principalement de la non prise en compte des effets visquetx puisque l'utilisation du code bidimensionnel avec le modète de pertes améliore les résultats. Cette sim- 


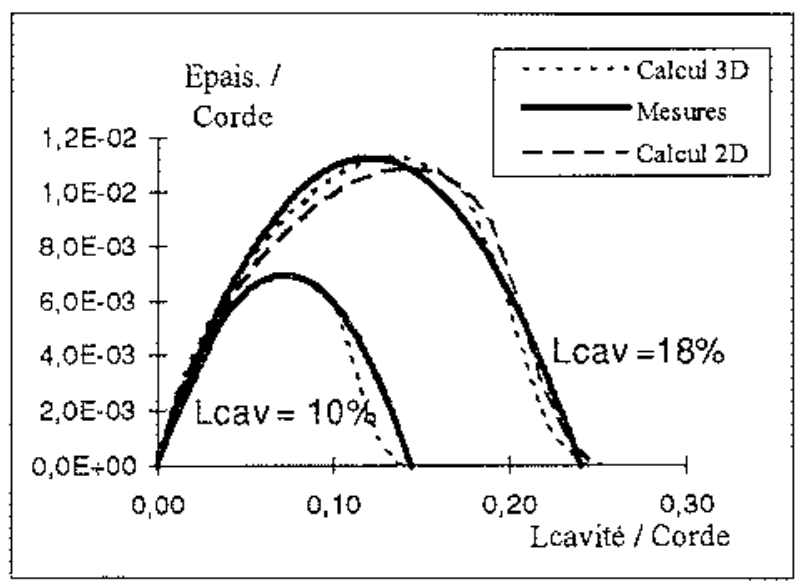

3. Comparaison des formes de poches.

plification du modèle est également à l'origine des fortes surpressions visibles sur les répartitions de coefficients de pression à l'aval du palicr de pression (effet classioue du sillage fermé). L'exploitation des courbes exprimant te sigme en fonction de la longueur de la cavité (figure 4) fait apparaître ce décalage entre les résułtats numériques et les mesures. Toutefois, lorsque l'on considère les pertes dans la veine à l'aide du modèle bidimensionnel on s'aperçoi! que la correspondance calcul-expérience en est rettement amélioréc. Cette dernière constatation, nous assure que les calcuis cavitants elfectues avec le code CALECHE-MOCA3D sont représentatifs de l'écoulcment réel.

\subsubsection{Discrétisation du bord d'attaque des aubages fins}

Si les variations de débit d'une machine hydraulique affectent les figures de cavilation qui se développent sur ses aubages, la définition géométrique des aubages, ci notamment de leur extrémité, cst un facteur déterminant. Stripling [8] dans un article consacré à la cavitation, fait éial d'un effet bénéfique, sur les performances cn cavilation, de l'affinage du bord d'allaque des aubages, notamment ayce une forme arrondic côté extrà̀os. On retrouve les mêmes tendances dans des articles de lforic et Ostima [9] et Moore el Meng [10]. Par conséquent, les résultats cxpérimentalix montrent clairement limportance d'une bonne description géométrique du bord d'attaque des anbages pour la modélisation des ćcoulements cavitants ; un maillage non adapté dans cette région (ne décrivant pas fídèlement la géométrie) peut fortcment perturber le calcul des cavités attachées aux aubages.

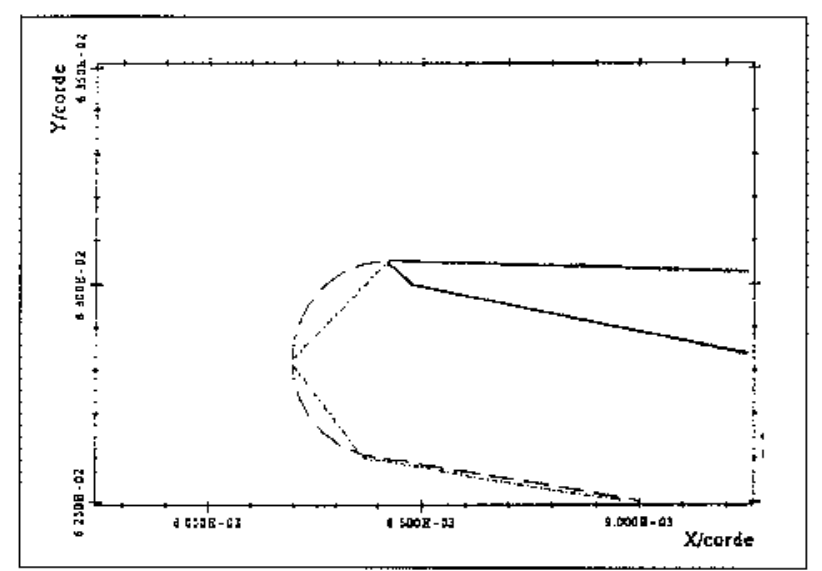

5. Les différentes géométries.

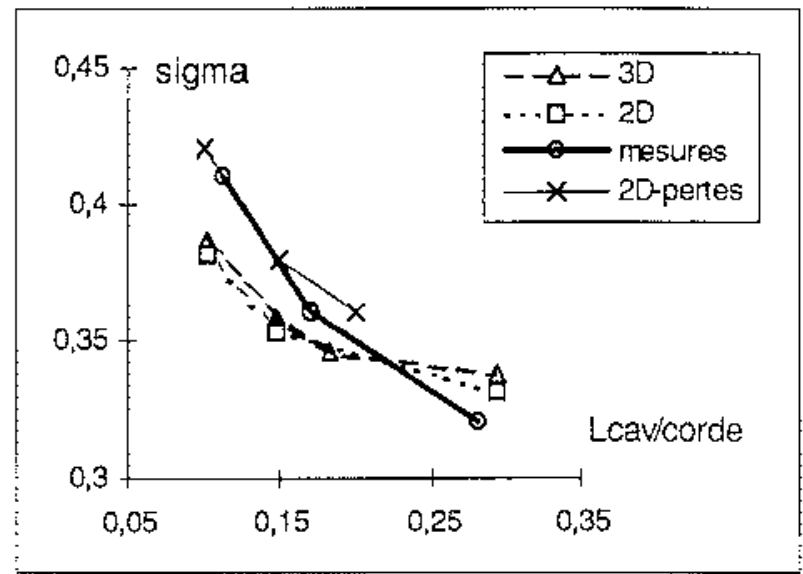

4. Comparaison des lois " $\sigma($ Leav $) "$.

L'objectif est de simplifier la discrétisation at niveau du bord d'attaque sans perturber les résultats des modélisations. Pour cela nous avons testé trois discrétisalions diflérentes (figure 5 ) en plus de celle qui considère l'arrondi d'usinage.

- Pour la géomélrie arrondic, le maillage compone 3036 ćléments dont une vingtaine répartie sur l'arondi ; la taille moyenne d'une maitle est done de l'ordre du dixième de millimètre. Le positionnement du point de détachement est effectué au moyen du critère du départ tangent (la poche se détache tangentiellement au profib). Remarọtons que ce critere est en accord avec une modélisation par interface ; cette dernière ne peut en aucun cas ĉtre fidèle à la géométric réclie de la cavité qui csi soumise à la tension superficielle.

- Le bord d'altaque triangulaire remplace larrondi par un triangie isocèle cont le sommet est placé au centre de l'arrondi. Chaq̣uc côté est défini à l'aide de deux mailtes, ce qui réduit le nombre déléments dans le sens amont-aval ce 18 . Le raccordement des faces dia triangle avec le profil provoque une arĉte vive qui sera le point de détachement des poches à l'extrados.

- Le bord d'attaque affinć correspond à une pointe "numériçue" (bord d'atlaque restreint à un seul noud qui appartient aussi bien à la première facette placćc à l'extrac̀os qu'aı premier élćment de l'inirados). Pour cela, le profil est affûté sur $10 \mathrm{~mm}$ soil $1 \%$ de la corde.

Les premiers calculs révèlent un comportement irréaliste pour lc bord d'attaque triangulaire. Co demier présente des angles trop importarts qui imposent un contournement exagéré nefaste ain couplage CALECHE-MOCA3D. Pour les pocines courtes, on

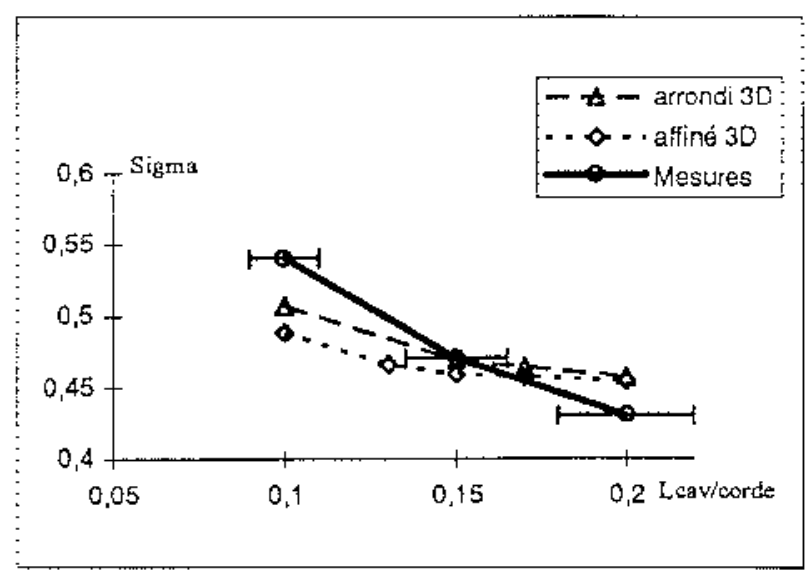

6. Loiss " $\sigma($ Lcav $) "$ str les différents bords d'attaques. 


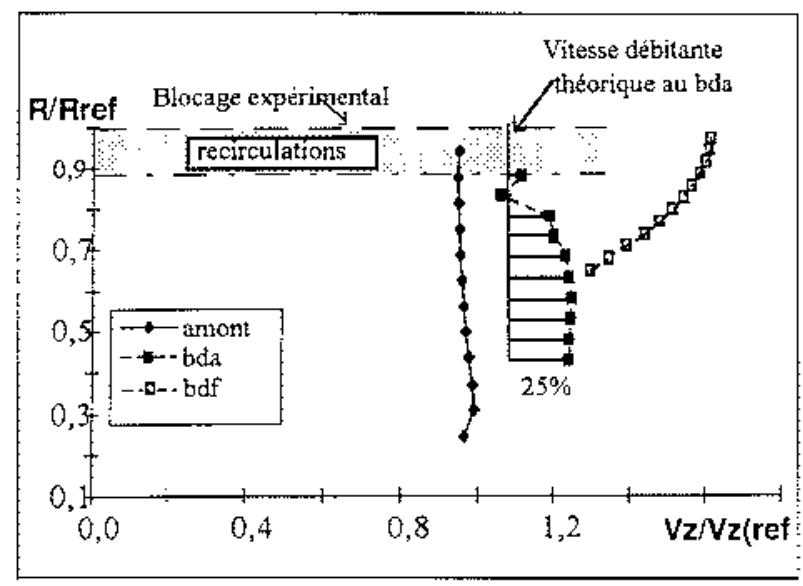

7. Profil de vitesses débitantes.

obscrve unc légère divergence entre les deux autres géométries (affrnée et arrondie) qui r'excède pas $5 \%$ sur le nombre de cavitation (figure 6). Pour une valeur dus sigma donnée, la poche atlachéc au prokil fin a tendance à être plus courte que celle observée sur l'arrondi, ce qui peut s'expliquer par ur contoumement moins fort du fluide et par conséguent des dépressions moins accentuées en non cavitant.

"L'affûtagc" du profil pour créer une pointe numérique niaffecte quasiment pas les résuliats. L'ulilisation des géométries de substitution, qui permettent de réduire sensiölement le raffinement du maillage au niveau du bord d'attaque et d'éliminer le problème du positionnement du point de détachement, est par conséquen envisageable pour les aubages fins. Dans ce cas, le nombre d'éléments du maillage a été réduit de $30 \%$, permellant d'économiser un temps de calcul du même ordre, ce qui est non négligeable pour les applications tridimensionnelles.

\section{IV - CALCUL D'LNE GÉOMETRIE TRIDIMENSIONNELLE}

Jusqqu'à présent je codic CALECHE-MOCA3D avait été utilisć pour prévoir, avec succès, les régimes cavitants de lurbomacrines présentant des aubages biseautés. Par cxemple, pour la denière machine calculée [11], le code CALECHE-MOCA3D prévoit, en régime subcavitant, une recirculation au niveau du bord d'attaque en tête de pale qui est en accorć avec les résuitals de calculs Navicr-Stokes, mais égatement avec les mesures par velocimétrie laser. Toutefois, le modète ćtant de type fluide par-

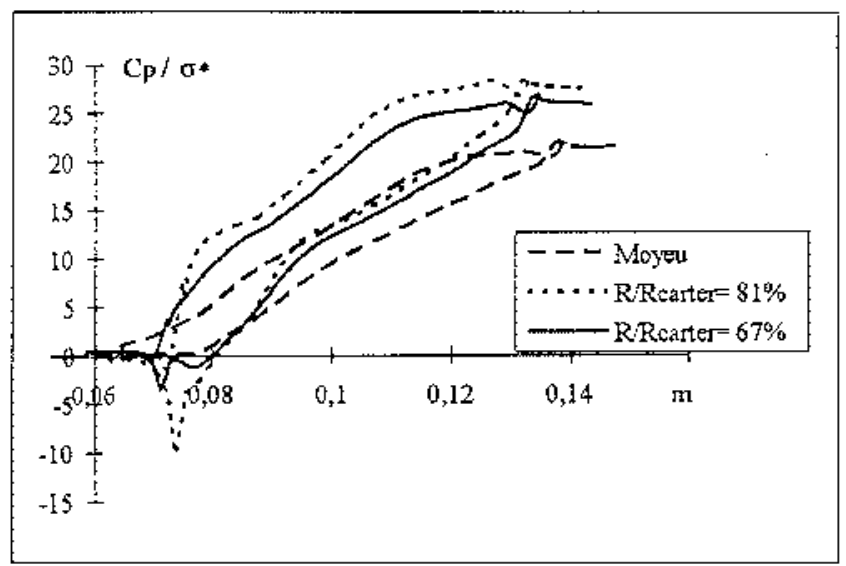

9. Répartitions de pression sur l'aubage.

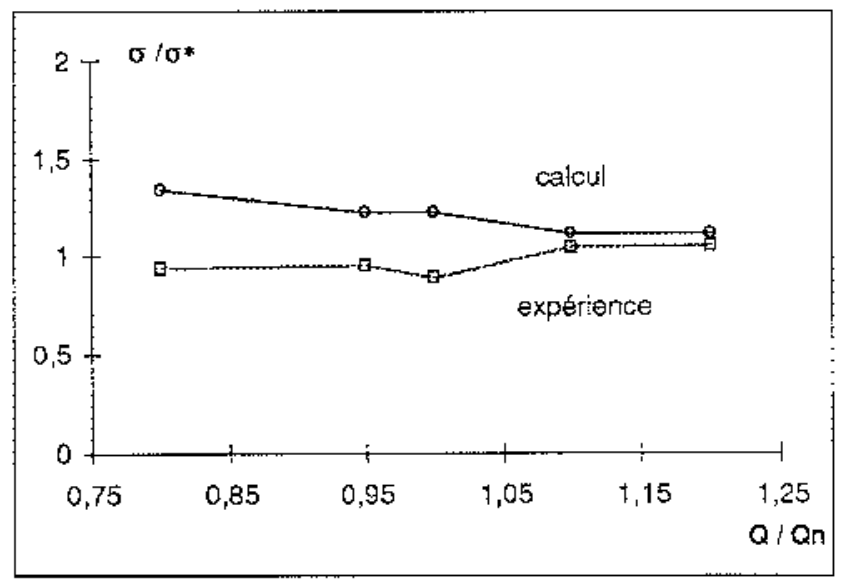

8. Comparaison des sigmas critinues calculés et mesurés.

fait, la recirculation est moins importante que dans la réalié. En effel, expérimentalement on mesure une réduction de $40 \%$ de la section ce passage débitante (augmentation de $40 \%$ ćgalement de la vitesse débitante dans la zonc non recirculante) aiors que dans notre caicul cette obstruction est de l'ordre de $25 \%$ (Figure 7). En régime caviant, la prévision des sigmas de blocage à diflérents débits (figure 8) est d'autant meilleure que les effets visqueux sont faibles ; en sur-débit les résultats numériques et cxpérimentaux sont très proches car l'écoulement présente moins de recirculations qu'en sous-détil.

A présent, notre objectif est de calculer les régimes cavitants d'une machine axiale, type pompe de gavage, qui présente des aubages fins mais avec un arrondti de $2 / 10^{\text {case }}$ Ge millimètre à leur extrémité. Le maillage d'un canal inter-aubes de celte machine est réalisé avec une grille $12 \times 22 \times 80$, soi 24219 nouds, en remplaçant I'arrondi d'usinage par une pointe $(\$ 3.2,2)$.

\subsection{Le régime non cavitant}

A l'issu du calcul en régime non cavitant, on relève (figure 9) un fort gradient ge pression statique entre l'cxtrados et l'intrados de l'aubage, notamment au niveau du carter. Notons égatement un fort decalage entre les nivezux de pression cn picd et cn tête de pale, avec des aubages beaucoup plus chargés en périphéric. Les importantes dépressions en tête d'aubage sont le signe d'unc apparition précoce de la cavitation.

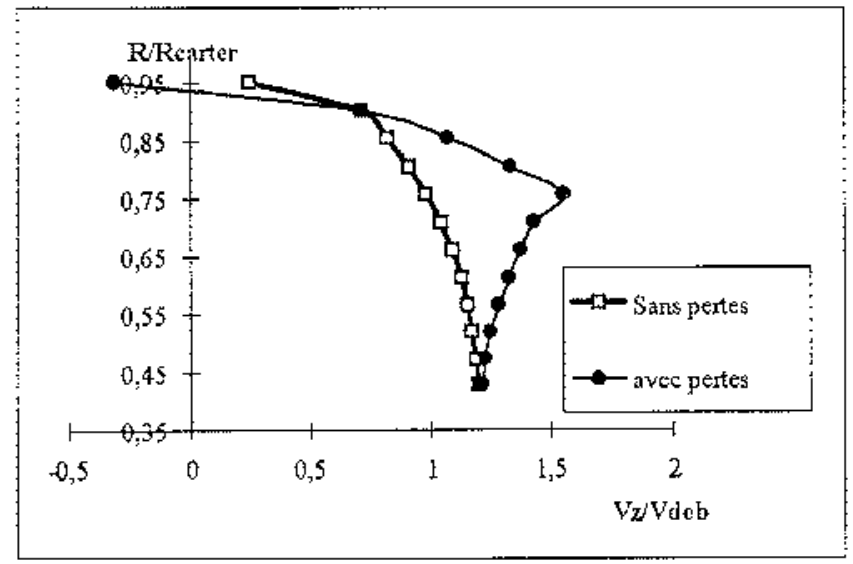

10. Vitesse débitante à l'entrée du canal inter-aubes. 


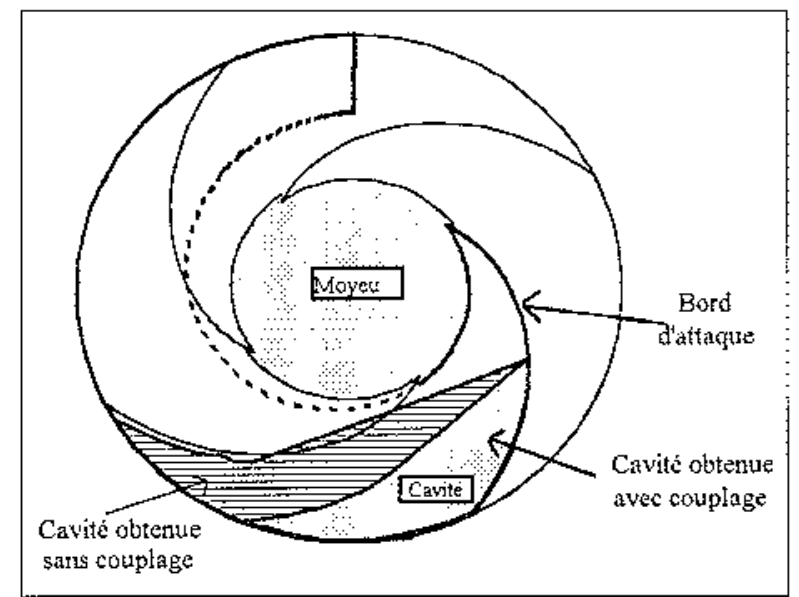

11. Vue axiale de la poche modélisée par le code CALECHE-MOCA3D.

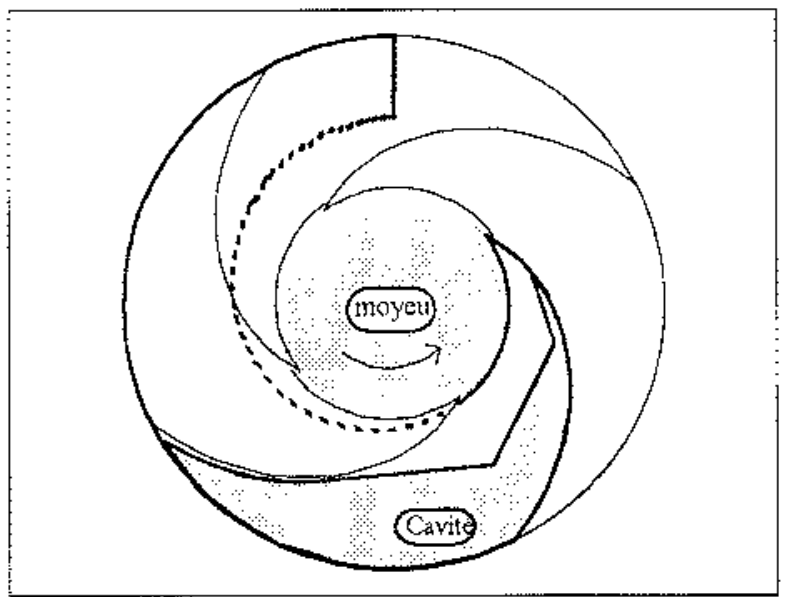

12. Forme de la poche modélisée par le code CALECHE-MOCA3D à $\sigma / \sigma^{*}=1,8$.
Expćrimentalement, cet écart de pression se traduit par la présence au carler d'un vortex cavitant à lous les régimes de tonctionrement. 'Toutefois, le fort gradient de pression entre l'extrados et l'intrados, qui favorise l'écoulement d'entrefer, doil jotser un rôle important dans l'écoulement au niveal de la centure ; $c t$ le fait de le négliger conduit probablement à une surestimation des gradients de pression moyet - carter dans notre caleul.

Pour connaître l'influence de la recirculation sur les modélisations cavitantes, nous avons utilisé une fonctionnalité du code CALECHE qui permet de prendire en compte des peres die pression totale relative. Par une distributior de pertes adaptée, on poura accrôtre lintensité ce la recirculation amont et se rapprociner de l'écoulement réel. Dans ce cas, te calcul montre clairement que l'écoulement contourne celle zone de basse pression, ce qui se traduit par des survitesses en dessous de la zone affectée par les pertes, et une réduction ce la vitesse dans la région où la pression totale relative est minimale (près du carter, figure 10). Avec cette distribution de pertes, nous avons done augmente l'intensité de la recireulation amont ct modifít l'incicence du farude à l'cntrée du canal : réduction de l'incidence dans la zone où la vitesse est augmentée ct augmentation de l'incidence près du carter. Par conséquent, l'utilisation de celte distribution contribue à une augmentation du gradicnt de pression moyeucarter.

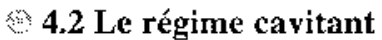

Lobjectis de ce calcul est de prédire les condilions de fonctionnement (notamment la pression en entrée) qui entraînent une chute de $3 \%$ des perlomances de la pompe. Le critère utilisé est celui détaitlé au $\$ 2.3$ : mocélisation d'une poche de caviation atteignant le recouvrement au moins on un point de :'albage.

Les résultats d'une étape de "prospecion" réalisée sans couplage CALECHE-MOCA3D confirment les principales constatations effectués à 'issue du calcul non cavitant, ì savoir l'apparition de la cavitation en périphérie pour des sigmas assez importanis. D'aulre part, on telève un comportement "explosif" de la poche de vapeur qui est traduit par des lois ơ(L), à différen:s rayons, assez plates. Par conséquent, une petite variation de la pression amont se tradtit par une forte variation de la longueus de poche. Ce caractère instable donne une importance particulière au couplage et à la procédure de descente en sigma. la figure 11 met en ćvidence l'intérêt de bien prenare en compte les effels tridimensionnels par le biais du couplage, sans quoi nous obtiendrions une surestimation de la longueur de la poche et du volume de vapeur.

Après une dizaine de couplages, soil cnviron une heure Cray, te code CAEECHE-MOCA3D prévoit une poche quit atteint le recouvrement sur la partie supéricure de l'aubage, pour une pression d'aspiration supéricure ì celle qui est obtenue expérimentalement $\left(\sigma / \sigma^{*}=1,8\right)$. Il ne prćwoil aucune cavitation près du moyeu (quart inféticur de fauhage), puis une poche de longueur très faible jassquà la mi-hauleur ; ensuite la longureur progresse régulièrement jusqu'au trois-quarts de la pate, et elle part brusquement au recouvrement sur le haut de la machine. On est done en présence d'une poche "triangulaire" (figure 12) dont le contour est similairc aux visuaijsaltions réalisées lors des essais. Cctte poche tridimensionnelle a un volume de $0,68 \%$ du volume inter-aubcs, ce qui restc assez faible mais proche du volume estimé par traitement dimages qui est de 0,6\%.

Notons que sur ce iype de machire, où les effets tridimensionnels sont assez importants, scule l'ulilisation d'un code tridimensionnel pest permette de modéliser une poche évoluant aussi fortement du moyeu au carter. On peut notamment soutigner que des calcuis faits sur cctte géométric par un code quasitridimensionnel :4] ont abouti à une poche homogène du moyeu au carter qui riest pas réaliste.

Malgré une bonne prévision de la forme de la cavité et du voíume de vapeur, le code ne prévoil pas correctement le régime critique de la machine; la poche atteignant le recouvrement est obtenue pour une pression d'aspiration supérieure à la pression mesurée expérimentalement. Cel ćcart peut provenir priacipalement de deux raisons

- Le critère de chute de performance n'est pas adapté à la machinc que nous venons de calculer. En effet, la poche ćtant principalement développéc en périphéric, elle s'alłonge peutctre dans le canal inter-aubes sans provoquer une chute des performances. 
- La deuxième raison serait une surestimation du gradient de pression moyeu-carter due à ia ron prise en comple de l'écoulement dentrefer ou des pertes sur les aubages. Dans ce cas, une distribution de pertes ćvoluant dans Ia direction azimutale peut permettre de modjicter l'incidence du fluide à l'entrée du canał et donc les dépressions.

\section{CONCLUSION}

Le calcul cavitan: cffectué avec le code CALECHE. MOCA3D sur cetle nouvelle gémétrie prévoil un développement non uniforme de la cavitation entre le moyeu et le carter, ce qui est en accord avec les visualisations. La forme de la poche est par conséquent fortement tridimensionnelle. La prévision du régime critique (apaissement de $3 \%$ de la hauteur manométricue) est pénaliséc par l'ultisation d'un critère purement géométrique (longueur de cuvité égale à la distance au col). Par conséquent, le développement d'an critère plus physique est envisagé, qui pourra porter soil sur la modification du triangle de vitesses en sortie di canal inter-aubes soit sur le couple.

\section{REMERCIEMENTS}

Les auteurs remercient la Sociétć Européenne de Propulsion et le Centre National dtetudes Spatiales pour le Financement de ces travaux, ainsi que toutes les personnes qui on: participé à celte étude. Les calculs on été réalisćs sur le Cray de l'institut du Développement et des Resscurces en Informatique Scientifique.

\section{BIBLIOGRAPHIE}

1: EL GHAZAN E.M. (1990), --.. Cileut ce lécoulement dans ste turteo.

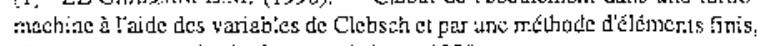
Thèse de doe:cur, lisule Centrate de Lyo: 1990

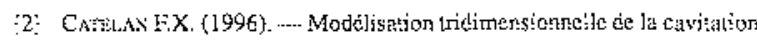

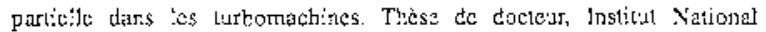
Po:ytechrigue de Grenoble 1596.

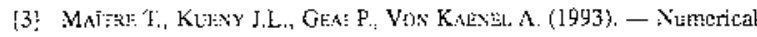
prediclicns of threedimensior.al pertal cay:tation is a rocket :urbopurnp inducer. Putrepirg Macininery Symposi:m, ASME, Wathinglon

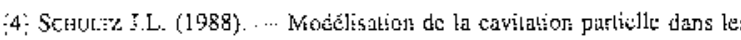
pompes. Thèse de doctedr, Institul Natjornt Polytectrique de Greroble 1988.

(5) Yangledil H., Karo H. (:983). - On app!:calion of ronitina cavily how theory to thick toil sections. Congnès IMilicit, and hiterr. Conf, on

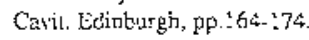

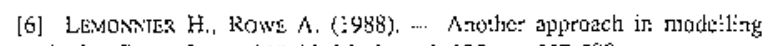

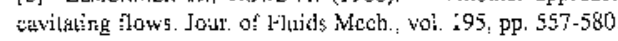

[7] REBOUJ J.'., Rlegate: C., Mrel. P. (1996). -- Elfec: of the teading edge cesign on shect cavitation around a blade section. XVlil IAHR symposium: HyǴraldic machitery and cavi:alivo, Valencia, Sept. 1996.

[8] S.RIJ.Xo L.B., ACosta A.J. (\$962). - Cavita:jon in lurbe pumps (part 1 et 2). Joumet of Bhsic Engigecring, scplember 1962.

[9] Honje C., Ostima M. (1954). - Experimentit sikfly on caviation in mixed thow pump: impeliers. Bulletin of JSME, vol. 7, $\mathrm{n}^{2} 25$, pp. 62-7ะ.

[:0: Moork D. Meisg P.R. (1970). - Elfect or bínde leiddirg edge thickness on cavialion performance of $80.6^{\circ}$ belint ind ineer in thydroger. NASA TN D. 5855 , june 1970

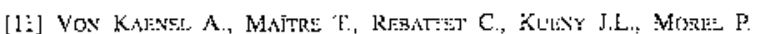

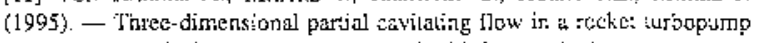

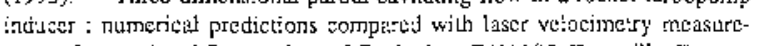
ments. Internationa' Symposium of Cavialion, CAV'95, Duan:I]c, France. 